\title{
Microwave-Assisted Synthesis of Some Quinoxaline-Incorporated Schiff Bases and Their Biological Evaluation
}

\author{
L. Achutha, R. Parameshwar, B. Madhava Reddy, and V. Harinadha Babu \\ Department of Pharmaceutical Chemistry, G. Pulla Reddy College of Pharmacy, Mehdipatnam, Hyderabad 500 028, India \\ Correspondence should be addressed to V. Harinadha Babu; parmi_pharma@yahoo.com
}

Received 4 December 2011; Accepted 9 May 2012

Academic Editor: Aleš Imramovsky

Copyright (C) 2013 L. Achutha et al. This is an open access article distributed under the Creative Commons Attribution License, which permits unrestricted use, distribution, and reproduction in any medium, provided the original work is properly cited.

\begin{abstract}
Quinoxaline-incorporated Schiff bases (4a-j) were synthesized by the condensation of 2-[(3-methylquinoxalin-2yl)oxy]acetohydrazide (3) with indole-3-carbaldehyde, furfuraldehyde, 5-(4-nitrophenyl)-2-furfuraldehyde, and substituted benzaldehydes under conventional and microwave irradiation methods. The microwave method was found to be remarkably successful with higher yields, less reaction time, and environmentally friendly compared to conventional heating method. The chemical structures of the synthesized compounds have been confirmed by analytical and spectral data. All the compounds have been evaluated for antitubercular and anti-inflammatory activities.
\end{abstract}

\section{Introduction}

Quinoxaline derivatives have been reported to possess a wide range of biological properties such as being anticancer $[1,2]$, antihistaminic [3], antiviral [4], antimicrobial [5, 6], antifungal [7], antitubercular [8,9], and anti-inflammatory [10]. Schiff bases possess antimicrobial [11], antitubercular [12], anti-inflammatory [13], and analgesic [14] properties. In view of these facts, we herein report the facile synthesis of a new series of quinoxaline-incorporated schiff bases and screen for antitubercular and anti-inflammatory activities. In recent times, microwave-assisted organic reactions have become very popular and gain special attention due to their ecofriendly nature, safety, less reaction time, and higher yields. In continuation of our research on quinoxaline derivatives, in the present study, we made an attempt to synthesize the title compounds using conventional and microwave irradiation methods and compared the reaction time and percentage yields.

\section{Experimental}

Melting points were determined using open capillary tubes on ANALAB melting point apparatus and are uncorrected. IR spectra were recorded on FTIR spectrophotometer (SHIMADZU 8400 series) using $\mathrm{KBr}$ pellets. ${ }^{1} \mathrm{H}$ NMR spectra were recorded in $\mathrm{CDCl}_{3} / \mathrm{DMSO}-\mathrm{d}_{6}$ solvents unless otherwise mentioned. Chemical shifts are reported on AVANCE $300 \mathrm{MHz}$ and INNOVA $400 \mathrm{MHz}$ relative to TMS internal standard on the $\delta$-scale. Mass spectra of the compounds were recorded on Mass spectrometer (Agilent 1100 series; EI/ESMS) at Indian Institute of Chemical Technology, Hyderabad. The syntheses were carried out in scientific microwave oven supplied by Catalyst, India. All the reactions were monitored by thin layer chromatography on $60 \mathrm{~F}_{254} 0.25 \mathrm{~mm}$ precoated aluminum plates (Merck) and visualized with UV light.

\section{Results and Discussion}

3-methylquinoxalin-2-ol (1) was synthesized by condensing o-phenylenediamine with ethyl pyruvate. Compound (1) was converted into ethyl [(3-methylquinoxalin-2-yl)oxy]acetate (2) by heating under reflux with ethyl chloroacetate in dry acetone and anhydrous potassium carbonate. The structure of compound (2) was confirmed by the carbonyl peak of ester at $1740 \mathrm{~cm}^{-1}$ in IR, and a mass ion peak at $\mathrm{m} / \mathrm{z} 247(100 \%)$ in mass spectroscopy. The reaction of the compound (2) with hydrazine hydrate (99\%) in ethanol under reflux for 5$6 \mathrm{hr}$ gave 2-[(3-methylquinoxaline-2-yl)oxy] acetohydrazide (3). The carbonyl peak of amide at $1680 \mathrm{~cm}^{-1}$ and $\mathrm{NH}, \mathrm{NH}_{2}$ stretching at $3330 \mathrm{~cm}^{-1}$ and $3226 \mathrm{~cm}^{-1}$ in IR and a mass 
<smiles>Nc1ccccc1N</smiles><smiles>CCOC(=O)C(C)=O</smiles><smiles>CC(C)[AsH2]Cc1nc2ccccc2nc1OCC(=O)N/N=C/[Al]</smiles>

$$
\begin{aligned}
& \mathbf{4 a}=\mathrm{Ar}=\mathrm{C}_{6} \mathrm{H}_{5} \\
& \mathbf{4} \mathbf{b}=\mathrm{Ar}=4-\mathrm{ClC}_{6} \mathrm{H}_{4} \\
& \mathbf{4} \mathbf{c}=\mathrm{Ar}=3-\mathrm{NO}_{2} \mathrm{C}_{6} \mathrm{H}_{4} \\
& \mathbf{4} \mathbf{d}=\mathrm{Ar}=4-\mathrm{OHC}_{6} \mathrm{H}_{4} \\
& \mathbf{4} \mathbf{e}=\mathrm{Ar}=3,4-\left(\mathrm{OCH}_{3}\right) \mathrm{C}_{6} \mathrm{H}_{3} \\
& \mathbf{4 f}=\mathrm{Ar}=4-\left(\mathrm{OCH}_{3}\right) \mathrm{C}_{6} \mathrm{H}_{4} \\
& \mathbf{4} \mathbf{g}=\mathrm{Ar}=4-\mathrm{N}\left(\mathrm{CH}_{3}\right)_{2} \mathrm{C}_{6} \mathrm{H}_{4} \\
& \mathbf{4 h}=\mathrm{Ar}=3 \text {-indolyl } \\
& \mathbf{4 i}=\mathrm{Ar}=2 \text {-furyl } \\
& \mathbf{4 j}=\mathrm{Ar}=5 \text {-(4-nitrophenyl)-2-furfuryl }
\end{aligned}
$$

SCHEME 1

ion peak at $\mathrm{m} / \mathrm{z} 233$ (100\%) corresponding to its molecular weight in mass spectrum confirmed the structure of the compound (3). From compound (3), ten different Schiff bases have been synthesized (Scheme 1 ) by condensing with indole-3-carbaldehyde, furfuraldehyde, 5-(4-nitrophenyl)-2furfuraldehyde, and substituted benzaldehydes using conventional and microwave irradiation methods. The physical data of Schiff bases, time and yields in both the methods, were compared and mentioned in the Table 1.

3.1. Antitubercular Activity. All the newly synthesized compounds were evaluated for their possible in vitro antitubercular activity at a concentration of $6.25 \mu \mathrm{g} / \mathrm{mL}$ against $M y c o b a c$ terium tuberculosis $H_{37} R V$ (ATCC 27294) in BACTEC 12B medium using both micro dilution assay and the microplate alamar blue assay (MABA). Compounds exhibiting fluorescence were tested in the BACTEC 460 radiometric system. The antitubercular activity data were compared with standard drug, Rifampin at a concentration of $0.25 \mu \mathrm{g} / \mathrm{mL}$. The compounds $\mathbf{4 c}, \mathbf{4 d}, \mathbf{4 i}$, and $\mathbf{4 j}$ exhibited substantial antitubercular activity, particularly $\mathbf{4 h}$ showed $75 \%$ inhibition
(MIC $>6.25 \mu \mathrm{g} / \mathrm{mL}$ ) and emerged as the most active compound in the present series. The MIC and percentage inhibition data of the compounds that have shown activity against Mycobacterium tuberculosis are presented in Table 2.

3.2. Anti-Inflammatory Activity. Male Wistar rats (140$200 \mathrm{~g}$ ) of both sexes were used for the studies. The rats were obtained from Mahaveer enterprises, Hyderabad. The animals were divided into groups of six each and fasted for $12 \mathrm{hr}$ before the experiment. The ethical guidelines prescribed for the investigation of animals used in experiments were followed in all tests.

Carrageenan $(0.1 \mathrm{~mL}, 1 \%)$ was administered into the plantar surface of the right hind paw of each rat, $1 \mathrm{hr}$ after the administration of the test compounds and standard drug ibuprofen $(100 \mathrm{mg} / \mathrm{kg})$. One group was kept as control, received only $0.5 \% \mathrm{CMC}$ solution. Before injection of carrageenan, the average volume $\left(V_{o}\right)$ of the right hind paw of each rat was calculated. After injection, the paw volume $\left(V_{t}\right)$ was measured after $3 \mathrm{rd}$ hr with the aid of a plethysmograph. The edema was expressed as an increase in the volume of paw, 
TABLE 1: Characterization data of compounds $\mathbf{4}(\mathbf{a}-\mathbf{j})$.

\begin{tabular}{|c|c|c|c|c|c|c|}
\hline \multirow[t]{2}{*}{ Comd } & \multirow[t]{2}{*}{$\mathrm{Ar}$} & \multirow[t]{2}{*}{ M.P $\left({ }^{\circ} \mathrm{C}\right)$} & \multirow{2}{*}{$\begin{array}{c}\text { Conventional } \\
\text { Time(hr) }\end{array}$} & \multicolumn{3}{|c|}{ Microwave } \\
\hline & & & & Yield (\%) & Time (min) & Yield (\%) \\
\hline $4 a$ & $\mathrm{C}_{6} \mathrm{H}_{5}$ & 278 & 6 & 75 & 7 & 90 \\
\hline $4 b$ & $4-\mathrm{Cl}-\mathrm{C}_{6} \mathrm{H}_{4}$ & 260 & 5 & 69 & 6 & 80 \\
\hline $4 c$ & $3-\mathrm{NO}_{2}-\mathrm{C}_{6} \mathrm{H}_{4}$ & 289 & 7 & 80 & 7 & 92 \\
\hline $4 d$ & $4-\mathrm{OH}-\mathrm{C}_{6} \mathrm{H}_{4}$ & 246 & 6 & 65 & 7 & 81 \\
\hline $4 e$ & $3,4-\left(\mathrm{OCH}_{3}\right)_{2}-\mathrm{C}_{6} \mathrm{H}_{3}$ & 240 & 6 & 76 & 8 & 85 \\
\hline $4 f$ & $4-\mathrm{OCH}_{3}-\mathrm{C}_{6} \mathrm{H}_{4}$ & 281 & 7 & 72 & 7 & 89 \\
\hline $4 g$ & $4-\mathrm{N}\left(\mathrm{CH}_{3}\right)_{2}-\mathrm{C}_{6} \mathrm{H}_{4}$ & 274 & 6 & 66 & 6 & 83 \\
\hline $4 h$ & 3-indolyl & 256 & 7 & 60 & 7 & 82 \\
\hline $4 i$ & 2-furyl & 295 & 5 & 68 & 6 & 84 \\
\hline $4 \mathbf{j}$ & 5-(4-nitrophenyl)-2-furfuryl & 232 & 6 & 74 & 8 & 91 \\
\hline
\end{tabular}

TABle 2: Percentage inhibition of compounds against Mycobacterium tuberculosis $\mathrm{H}_{37} R V$ at MIC $(6.25 \mu \mathrm{g} / \mathrm{mL}) \mathbf{4}(\mathbf{a}-\mathbf{j})$.

\begin{tabular}{lc}
\hline Comd & \% Inhibition \\
\hline $\mathbf{4 a}$ & 23 \\
$\mathbf{4 b}$ & 15 \\
$\mathbf{4 c}$ & 66 \\
$\mathbf{4 d}$ & 58 \\
$\mathbf{4 e}$ & 25 \\
$\mathbf{4 f}$ & 10 \\
$\mathbf{4 g}$ & 32 \\
$\mathbf{4 h}$ & 75 \\
$\mathbf{4 i}$ & 56 \\
$\mathbf{4 j}$ & 50 \\
Rifampin & 95 \\
\hline
\end{tabular}

and percentage inhibition of acute edema was obtained as follows:

$$
\% \text { Inhibition }=\left[1-\left(\frac{\Delta V \text { experimental }}{\Delta V \text { control }}\right)\right] \times 100
$$

where

$$
\Delta V=V_{t}-V_{o}=\text { Mean paw volume. }
$$

Among all the compounds, compounds 4a, 4b, 4d, 4g, 4h and $4 \mathbf{i}$ exhibited significant activity at $3 \mathrm{rd} \mathrm{hr}$ (Table 3 ). Results are presented as Mean \pm SEM (standard error of mean) of six rats. Statistical analysis was performed using one-way analysis of variance (ANOVA) followed by Dunnett's test for multiple comparisons, using Graph-pad Software.

3.3. Toxicity Study. Acute toxicity of schiff bases was determined in albino mice with the staircase method. Each group of 5 animals was fasted for $24 \mathrm{hr}$ prior to the administration of the test compounds. The test compounds, $\mathbf{4 a}, \mathbf{4 b}, \mathbf{4 d}, \mathbf{4 g}, \mathbf{4 h}$
TABLE 3: In vivo anti-inflammatory activities of Schiff bases.

\begin{tabular}{lcc}
\hline Comd & $\begin{array}{c}V_{t}-V_{0} \\
{[\text { Mean } \pm \text { SEM }]}\end{array}$ & $\begin{array}{c}\text { \% Inhibition of edema at } \\
\text { the end of 3rd hr }\end{array}$ \\
\hline $\mathbf{4 a}$ & $0.42 \pm 0.011$ & 55.3 \\
$\mathbf{4 b}$ & $0.32 \pm 0.004$ & 65.9 \\
$\mathbf{4 d}$ & $0.38 \pm 0.005$ & 59.5 \\
$\mathbf{4 g}$ & $0.34 \pm 0.003$ & 63.8 \\
$\mathbf{3 h}$ & $0.33 \pm 0.004$ & 64.8 \\
$\mathbf{4 i}$ & $0.32 \pm 0.004$ & 65.9 \\
Ibuprofen & $0.26 \pm 0.018$ & 73.5 \\
\hline
\end{tabular}

Values expressed as Mean $\pm \mathrm{SEM}, n=6$ in each group $P>0.01$ compared with control.

and $4 \mathbf{i}$ were administered orally in doses up to $2000 \mathrm{mg} / \mathrm{kg}$ and mice were kept under observation for period of $24 \mathrm{hrs}$.

\section{General Procedure for the Synthesis of Schiff Bases}

4.1. Microwave Irradiation Method. 2-[(3-Methylquinoxalin2-yl)oxy] acetohydrazide (3) (0.01 mole, $2.32 \mathrm{~g}$ ) was dissolved in $10 \mathrm{~mL}$ of DMF and to this was added heterocyclic/aromatic aldehyde ( 0.01 mole) and few drops of glacial acetic acid. The mixture was transferred to a vessel and kept in the microwave oven. The oven was run at $400-480 \mathrm{~W}$ for different time for different reaction mixtures. The completion of the reaction was monitored continuously by TLC after every minute. The product obtained was poured into water, filtered, dried, and recrystallized from a mixture of DMF and water.

4.2. Conventional Method. Equimolar mixture of 2-[(3methylquinoxalin-2-yl)oxy]acetohydrazide (3) and appropriate heterocyclic/aromatic aldehyde was dissolved in $90 \%$ ethanol and to this was added catalytic amount of glacial acetic acid and refluxed for 5-7 hr. Excess ethanol was removed under reduced pressure, and the residue was purified by washing with cold ethanol and recrystallized from DMF and water mixture. 
2-[(3-Methylquinoxalin-2-yl)oxy]-N'-[(1E)-phenylmethylene]acetohydrazide 4a. Colorless solid; IR (KBr): 1656.74 ( $\mathrm{C}=\mathrm{N}$ str), 1679.88 ( $\mathrm{C}=\mathrm{O}$ str), 2958.6 (C-H str of $\left.\mathrm{CH}_{3}\right) ;{ }^{1} \mathrm{H}$ NMR $\left(\mathrm{CDCl}_{3}\right): \delta 11.84(\mathrm{~s}, 1 \mathrm{H}, \mathrm{NH}), 8.08(\mathrm{~s}$, $1 \mathrm{H}, \mathrm{CH}=\mathrm{N}), 7.77-7.36(\mathrm{~m}, 9 \mathrm{H}, \mathrm{Ar}-\mathrm{H}), 5.46\left(\mathrm{~s}, 2 \mathrm{H}, \mathrm{OCH}_{2}\right)$, $2.48\left(\mathrm{~s}, 3 \mathrm{H}, \mathrm{CH}_{3}\right)$; Mass $(\mathrm{M}+\mathrm{H})^{+}: \mathrm{m} / \mathrm{z} 321$ (100\%). Anal. Calcd for $\mathrm{C}_{18} \mathrm{H}_{16} \mathrm{~N}_{4} \mathrm{O}_{2}$ : C, 67.49; H, 5.03; N, 17.49. Found: C, $67.33 ; \mathrm{H}, 5.01 ; \mathrm{N}, 17.32 \%$.

$N^{\prime}$-[(1E)-(4-Chlorophenyl)methylene]-2-[(3-methylquinoxalin-2-l)oxy] acetohydrazide 4b. Colorless solid; IR (KBr): 1654.81 ( $\mathrm{C}=\mathrm{N}$ str), 1685.67 ( $\mathrm{C}=\mathrm{O}$ str), 2958.6 (C-H str of $\left.\mathrm{CH}_{3}\right), 754.12(\mathrm{C}-\mathrm{Cl}) ;{ }^{1} \mathrm{H}$ NMR $\left(\mathrm{CDCl}_{3}\right): \delta 11.92(\mathrm{~s}, 1 \mathrm{H}$, $\mathrm{NH}), 8.23$ (s, 1H, CH=N), 7.89-7.47 (m, 8H, Ar-H), 5.54 $\left(\mathrm{s}, 2 \mathrm{H}, \mathrm{OCH}_{2}\right), 2.81\left(\mathrm{~s}, 3 \mathrm{H}, \mathrm{CH}_{3}\right) ;$ Mass $(\mathrm{M}+\mathrm{H})^{+}: \mathrm{m} / \mathrm{z} 355$ (100\%). Anal. Calcd for $\mathrm{C}_{18} \mathrm{H}_{15} \mathrm{Cl} \mathrm{N}_{4} \mathrm{O}_{2}$ : C, 60.94; H, 4.26; N, 15.79. Found: C, 60.73; H, 4.15; N, 15.74\%.

2-[(3-Methylquinoxalin-2-yl)oxy]-N -[(1E)-(3-nitrophenyl)methylene]acetohydrazide 4c. Pale yellow solid; IR (KBr): 1654.81 (C=N str), 1689.53 (C=O str), 2948.96 (C-H str of $\left.\mathrm{CH}_{3}\right), 1320,1480\left(\mathrm{NO}_{2}\right.$ str $) ;{ }^{1} \mathrm{H} \mathrm{NMR}\left(\mathrm{CDCl}_{3}\right): \delta 11.96$ (s, 1H, NH), $8.42(\mathrm{~s}, 1 \mathrm{H}, \mathrm{CH}=\mathrm{N}), 7.98-7.53(\mathrm{~m}, 8 \mathrm{H}, \mathrm{Ar}-\mathrm{H})$, $5.67\left(\mathrm{~s}, 2 \mathrm{H}, \mathrm{OCH}_{2}\right), 2.92\left(\mathrm{~s}, 3 \mathrm{H}, \mathrm{CH}_{3}\right) ;$ Mass $(\mathrm{M}+\mathrm{H})^{+}: \mathrm{m} / \mathrm{z}$ 366 (100\%). Anal. Calcd for $\mathrm{C}_{18} \mathrm{H}_{15} \mathrm{~N}_{5} \mathrm{O}_{4}$ : C, 59.18; H, 4.14; N, 19.17. Found: C, 59.13; H, 4.11; N, 19.12\%.

$N$-[(1E)-(4-Hydroxyphenyl)methylene]-2-[(3-methylquinoxalin-2-yl)oxy] acetohydrazide 4d. Colorless solid; IR (KBr): 1658.67 (C=N str), 1681.81 (C=O str), 2958.6 (C-H str of $\left.\mathrm{CH}_{3}\right), 3352.05\left(\mathrm{O}-\mathrm{H}\right.$ str); ${ }^{1} \mathrm{H}$ NMR $\left(\mathrm{CDCl}_{3}\right): \delta 11.64$ $(\mathrm{d}, J=10.2, \mathrm{NH}), 9.94(\mathrm{~s}, 1 \mathrm{H}, \mathrm{OH}), 7.97(\mathrm{~s}, 1 \mathrm{H}, \mathrm{N}=\mathrm{CH})$, 7.78-6.83 (m, 8H, Ar-H), $5.41\left(\mathrm{~s}, 2 \mathrm{H}, \mathrm{OCH}_{2}\right), 2.47$ (s, 3H, $\left.\mathrm{CH}_{3}\right)$; Mass $(\mathrm{M}+\mathrm{H})^{+}: \mathrm{m} / \mathrm{z} 337(100 \%)$. Anal. Calcd for $\mathrm{C}_{18} \mathrm{H}_{16} \mathrm{~N}_{4} \mathrm{O}_{3}$ : C, 64.28; H, 4.79; N, 16.66. Found: C, 64.13; H, $4.71 ; \mathrm{N}, 16.56 \%$.

$N$-[(1E)-(3,4-Dimethoxyphenyl)methylene]-2-[(3-methylquinoxalin-2-yl)oxy]acetohydrazide 4e. Pale brown solid; IR (KBr): 1647.1 (C=N str), 1677.95 (C=O str), 2948.96 (C-H str of $\left.\mathrm{CH}_{3}\right), 1267.14$ (C-O-C str); ${ }^{1} \mathrm{H}$ NMR $\left(\mathrm{CDCl}_{3}\right)$ : $\delta 11.23(\mathrm{~s}, 1 \mathrm{H}, \mathrm{NH}), 8.13(\mathrm{~s}, 1 \mathrm{H}, \mathrm{CH}=\mathrm{N}), 7.83-7.47(\mathrm{~m}, 7 \mathrm{H}$, $\mathrm{Ar}-\mathrm{H}), 5.48\left(\mathrm{~s}, 2 \mathrm{H}, \mathrm{OCH}_{2}\right), 3.82\left(\mathrm{~s}, 6 \mathrm{H}, \mathrm{OCH}_{3}\right), 2.43(\mathrm{~s}$, $\left.3 \mathrm{H}, \mathrm{CH}_{3}\right)$; Mass $(\mathrm{M}+\mathrm{H})^{+}: \mathrm{m} / \mathrm{z} 381(100 \%)$. Anal. Calcd for $\mathrm{C}_{20} \mathrm{H}_{20} \mathrm{~N}_{4} \mathrm{O}_{4}: \mathrm{C}, 63.15 ; \mathrm{H}, 5.30 ; \mathrm{N}, 14.73$. Found: C, 63.07; H, $5.28 ; \mathrm{N}, 14.62 \%$.

$N$-[(1E)-(4-Methoxyphenyl)methylene]-2-[(3-meth-

ylquinoxalin-2-yl)oxy] acetohydrazide 4 f. Colorless solid; IR (KBr): 1654.81 (C=N str), 1679.88 (C=O str), 2962.46 (C-H str of $\left.\mathrm{CH}_{3}\right), 1257.50\left(\mathrm{C}-\mathrm{O}-\mathrm{C}\right.$ str); ${ }^{1} \mathrm{H}$ NMR $\left(\mathrm{CDCl}_{3}\right): \delta$ 11.53 (s, $1 \mathrm{H}, \mathrm{NH}), 8.24(\mathrm{~s}, 1 \mathrm{H}, \mathrm{CH}=\mathrm{N}), 7.82-7.58(\mathrm{~m}, 8 \mathrm{H}$, $\mathrm{Ar}-\mathrm{H}), 5.53\left(\mathrm{~s}, 2 \mathrm{H}, \mathrm{OCH}_{2}\right), 3.88\left(\mathrm{~s}, 3 \mathrm{H}, \mathrm{OCH}_{3}\right), 2.51$ (s, $\left.3 \mathrm{H}, \mathrm{CH}_{3}\right)$; Mass $(\mathrm{M}+\mathrm{H})^{+}: \mathrm{m} / \mathrm{z} 351(100 \%)$. Anal. Calcd for $\mathrm{C}_{19} \mathrm{H}_{18} \mathrm{~N}_{4} \mathrm{O}_{3}$ : C, 65.13; H, 5.18; N, 15.99. Found: C, 65.10; H, $5.06 ; \mathrm{N}, 15.63 \%$.

N-(1E)-[4-(Dimethylamino)phenyl]methylene-2-[(3methylquinoxalin-2-yl)oxy] acetohydrazide 4g. Colorless solid; IR ( $\mathrm{KBr}$ ): 1654.81 (C=N str), 1677.95 (C=O str), 2920 $\left(\mathrm{C}-\mathrm{H}\right.$ str of $\left.\mathrm{CH}_{3}\right), 1365.51$ (C-N str); ${ }^{1} \mathrm{H}$ NMR $\left(\mathrm{CDCl}_{3}\right): \delta$ 11.16 (s, 1H, NH), 8.15 (s, $1 \mathrm{H}, \mathrm{CH}=\mathrm{N}), 7.75-7.38(\mathrm{~m}, 7 \mathrm{H}$, $\mathrm{Ar}-\mathrm{H}), 5.32\left(\mathrm{~s}, 2 \mathrm{H}, \mathrm{OCH}_{2}\right), 3.19\left(\mathrm{~s}, 6 \mathrm{H}, \mathrm{N}\left(\mathrm{CH}_{3}\right)_{2}\right), 2.32$ (s, $\left.3 \mathrm{H}, \mathrm{CH}_{3}\right)$; Mass $(\mathrm{M}+\mathrm{H})^{+}: \mathrm{m} / \mathrm{z} 364(100 \%)$. Anal. Calcd for
$\mathrm{C}_{20} \mathrm{H}_{21} \mathrm{~N}_{5} \mathrm{O}_{2}$ : C, 66.10; $\mathrm{H}, 5.82 ; \mathrm{N}, 19.27$. Found: C, 66.05; $\mathrm{H}$, 5.66 ; N, 19.23\%.

$N$-[(1E)-1H-Indol-3-ylmethylene]-2-[(3-methylquinoxalin-2-yl)oxy]acetohydrazide 4 h. Pale brown solid; IR (KBr): 1650.95 (C=N str), 1677.95 (C=O str), 2991.39 $\left(\mathrm{C}-\mathrm{H}\right.$ str of $\left.\mathrm{CH}_{3}\right), 3263.33\left(\mathrm{~N}-\mathrm{H}\right.$ str of Ind-NH); ${ }^{1} \mathrm{H}$ NMR $\left(\mathrm{CDCl}_{3}\right): \delta 11.58(\mathrm{~d}, J=19.8,2 \mathrm{H}$, Ind-H, NH), $8.268(\mathrm{~s}, 1 \mathrm{H}$, $\mathrm{CH}=\mathrm{N}), 8.17-7.15(\mathrm{~m}, 9 \mathrm{H}, \mathrm{Ar}-\mathrm{H}), 5.5\left(\mathrm{~s}, 2 \mathrm{H}, \mathrm{OCH}_{2}\right), 2.47$ $\left(\mathrm{s}, 3 \mathrm{H}, \mathrm{CH}_{3}\right)$; Mass $(\mathrm{M}+\mathrm{H})^{+}: \mathrm{m} / \mathrm{z} 360$ (100\%). Anal. Calcd for $\mathrm{C}_{20} \mathrm{H}_{17} \mathrm{~N}_{5} \mathrm{O}_{7}$ : C, 66.84; $\mathrm{H}, 4.77 ; \mathrm{N}, 19.49$. Found: C, 66.75; $\mathrm{H}, 4.66$; N, 19.39\%.

$N^{\prime}$-[(1E)-2-Furylmethylene]-2-[(3-methylquinoxalin-2yl)oxy] acetohydrazide 4i. Pale yellow solid; IR (KBr): 1660.6 (C=N str), $1683.74\left(\mathrm{C}=\mathrm{O}\right.$ str), $2954.74\left(\mathrm{C}-\mathrm{H}\right.$ str of $\left.\mathrm{CH}_{3}\right)$, 1288.36 (C-O str); ${ }^{1} \mathrm{H}$ NMR $\left(\mathrm{CDCl}_{3}\right): \delta 11.77(\mathrm{~d}, J=7.6,1 \mathrm{H}$, $\mathrm{NH}), 7.96(\mathrm{~s}, 1 \mathrm{H}, \mathrm{CH}=\mathrm{N}), 7.84-6.64(\mathrm{~m}, 7 \mathrm{H}, \mathrm{Ar}-\mathrm{H}), 5.36(\mathrm{~s}$, $\left.2 \mathrm{H}, \mathrm{OCH}_{2}\right), 2.46\left(\mathrm{~s}, 3 \mathrm{H}, \mathrm{CH}_{3}\right) ;$ Mass $(\mathrm{M}+\mathrm{H})^{+}: \mathrm{m} / \mathrm{z} 311$ (100\%). Anal. Calcd for $\mathrm{C}_{16} \mathrm{H}_{14} \mathrm{~N}_{4} \mathrm{O}_{3}: \mathrm{C}, 61.93 ; \mathrm{H}, 4.55 ; \mathrm{N}$, 18.06. Found: C, 61.85; H, 4.66; N, 18.35\%.

2-[(3-Methylquinoxalin-2-yl)oxy]-N' -(1E)-[5-(4-nitrophenyl)-2-furfuryl]methyleneacetohydrazide 4j. Yellow solid; IR (KBr): 1654.61 ( $\mathrm{C}=\mathrm{N}$ str), 1689.4 ( $\mathrm{C}=\mathrm{O}$ str), 2956.24 $\left(\mathrm{C}-\mathrm{H}\right.$ str of $\left.\mathrm{CH}_{3}\right), 1286.53\left(\mathrm{C}-\mathrm{O}\right.$ str); ${ }^{1} \mathrm{H}$ NMR $\left(\mathrm{CDCl}_{3}\right)$ : $\delta 11.69(\mathrm{~s}, 1 \mathrm{H}, \mathrm{NH}), 7.84(\mathrm{~s}, 1 \mathrm{H}, \mathrm{CH}=\mathrm{N}), 7.78-6.73(\mathrm{~m}$, $10 \mathrm{H}, \mathrm{Ar}-\mathrm{H}), 5.24$ (s, $\left.2 \mathrm{H}, \mathrm{OCH}_{2}\right), 2.53$ (s, 3H, $\mathrm{CH}_{3}$ ); Mass $(\mathrm{M}+\mathrm{H})^{+}: \mathrm{m} / \mathrm{z} 432(100 \%)$. Anal. Calcd for $\mathrm{C}_{22} \mathrm{H}_{17} \mathrm{~N}_{5} \mathrm{O}_{5}: \mathrm{C}$, 61.25; H, 3.97; N, 16.23. Found: C, 61.18; H, 3.68; N, 16.15\%.

\section{Conclusion}

A novel series of schiff bases were synthesized by using conventional and microwave irradiation methods and all the compounds were characterized by physical and spectral data. Compounds $\mathbf{4 c}, \mathbf{4 d}, \mathbf{4 h}, \mathbf{4 i}$, and $\mathbf{4 j}$ exhibited potent antitubercular activity. Compounds $4 \mathbf{a}, \mathbf{4 b}, \mathbf{4 d}, \mathbf{4 g}, \mathbf{4 h}$, and $4 \mathbf{i}$ have shown significant protection against the edema formation. With further molecular modification and manipulation of these compounds, several other promising bioactive molecules can be developed in future.

\section{Acknowledgments}

The authors are thankful to the Management of G. Pulla Reddy College of Pharmacy, Hyderabad, for providing facilities. Thanks are also due to IICT and NATCO Pharma Ltd., Hyderabad, for providing Mass and NMR spectral data.

\section{References}

[1] A. Carta, M. Loriga, S. Zanetti, and L. A. Sechi, "Quinoxalin2-ones: Part 5. Synthesis and antimicrobial evaluation of 3alkyl-, 3-halomethyl- and 3-carboxyethylquinoxaline-2-ones variously substituted on the benzo-moiety," Farmaco, vol. 58, no. 12, pp. 1251-1255, 2003.

[2] H. Lee, S. Cho, K. Namgoong, J. K. Jung, J. Cho, and S. I. Yang, "Synthesis and in vitro evaluation of 7-dialkylaminomethylbenzo[g] quinoxaline-5,10-diones," Bioorganic and Medicinal Chemistry Letters, vol. 14, no. 5, pp. 1235-1237, 2004. 
[3] C. H. Sridevi, K. Balaji, A. Naidu, and R. Sudhakaran, "Synthesis of some Phenylpyrazolo benzimidazolo quinoxaline derivatives as potent antihistaminic agents," E-Journal of Chemistry, vol. 7, no. 1, pp. 234-238, 2010.

[4] N. Patel, J. Bergman, and A. Graslund, "1H-NMR studies of the interaction between a self-complementary deoxyoligonucleotide duplex and indolo[2,3-b]quinoxalone derivatives active against herpes virus," European Journal of Biochemistry, vol. 197, no. 3, pp. 597-604, 1991.

[5] H. M. Refaat, A. A. Moneer, and O. M. Khalil, "Synthesis and antimicrobial activity of certain novel quinoxalines," Archives of Pharmacal Research, vol. 27, no. 11, pp. 1093-1098, 2004.

[6] V. K. Tandon, D. B. Yadav, H. K. Maurya, A. K. Chaturvedi, and P. K. Shukla, "Design, synthesis, and biological evaluation of 1,2,3-trisubstituted-1,4-dihydrobenzo[g]quinoxaline5,10-diones and related compounds as antifungal and antibacterial agents," Bioorganic and Medicinal Chemistry, vol. 14, no. 17, pp. 6120-6126, 2006.

[7] Y. Kurasawa, A. Tsuruoka, N. Rikiishi, N. Fujiwara, Y. Okamoto, and H. S. Kim, "Quinolone analogues 1. A convenient synthesis of 1-alkyl-4-oxo-1,4-dihydropyridazino[3,4-b]quinoxaline3-carboxylic acids," Journal of Heterocyclic Chemistry, vol. 37, no. 4, pp. 791-798, 2000.

[8] E. Vicente, P. R. Duchowicz, E. A. Castro, and A. Monge, "QSAR analysis for quinoxaline-2-carboxylate 1,4-di-N-oxides as anti-mycobacterial agents," Journal of Molecular Graphics and Modelling, vol. 28, no. 1, pp. 28-36, 2009.

[9] A. Jaso, B. Zarranz, I. Aldana, and A. Monge, "Synthesis of new 2-acetyl and 2-benzoyl quinoxaline 1,4-di-N-oxide derivatives as anti-Mycobacterium tuberculosis agents," European Journal of Medicinal Chemistry, vol. 38, no. 9, pp. 791-800, 2003.

[10] Asunción Burguete, Eleni Pontiki, Dimitra Hadjipavlou-Litina et al., "Synthesis and anti-inflammatory/antioxidant activities of some new ring substituted 3-phenyl-1-(1,4-di-N-oxide quinoxalin-2-yl)-2-propen-1-one derivatives and of their 4,5dihydro-(1H)-pyrazole analogues," Bioorganic and Medicinal Chemistry Letters, vol. 17, no. 23, pp. 6439-6443, 2007.

[11] S. J. Wadher, N. A. Karande, S. D. Sonawane, and P. G. Yeole, "Synthesis and biological evaluation of schiff base and 4-thiazolidinones of amino salicylic acid and their derivatives as an antimicrobial agent," International Journal of ChemTech Research, vol. 1, no. 4, pp. 1303-1307, 2009.

[12] M. J. Hearn and M. H. Cynamon, "Design and synthesis of antituberculars: preparation and evaluation against Mycobacterium tuberculosis of an isoniazid Schiff base," Journal of Antimicrobial Chemotherapy, vol. 53, no. 2, pp. 185-191, 2004.

[13] G. Rajitha, N. Saideepa, and P. Praneetha, "Synthesis and evaluation of $\mathrm{N}$-( $\alpha$-benzamido cinnamoyl) aryl hydrazone derivatives for anti-inflammatory and antioxidant activities," Indian Journal of Chemistry B, vol. 50, no. 5, pp. 729-733, 2011.

[14] S. M. Sondhi, M. Dinodia, S. Jain, and A. Kumar, "Synthesis of biologically active novel bis Schiff bases, bis hydrazone and bis guanidine derivatives," Indian Journal of Chemistry B, vol. 48, no. 8, pp. 1128-1136, 2009. 

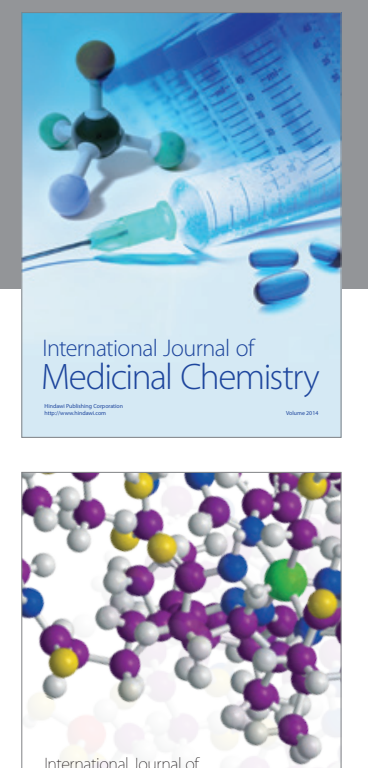

\section{Carbohydrate} Chemistry

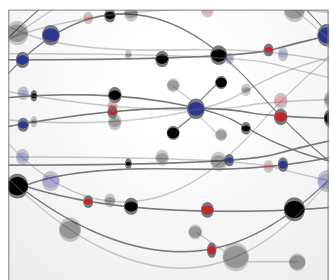

The Scientific World Journal
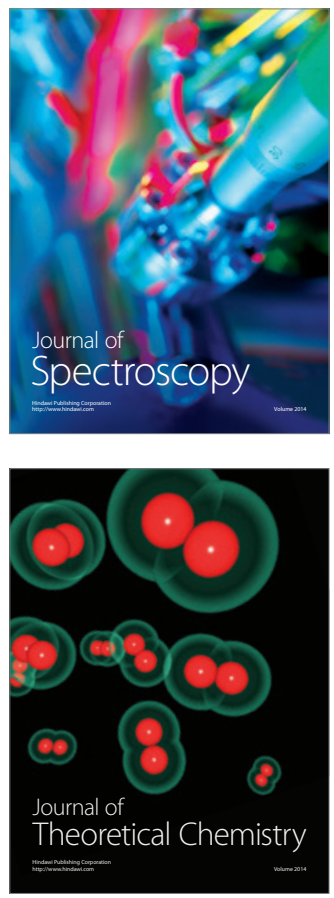
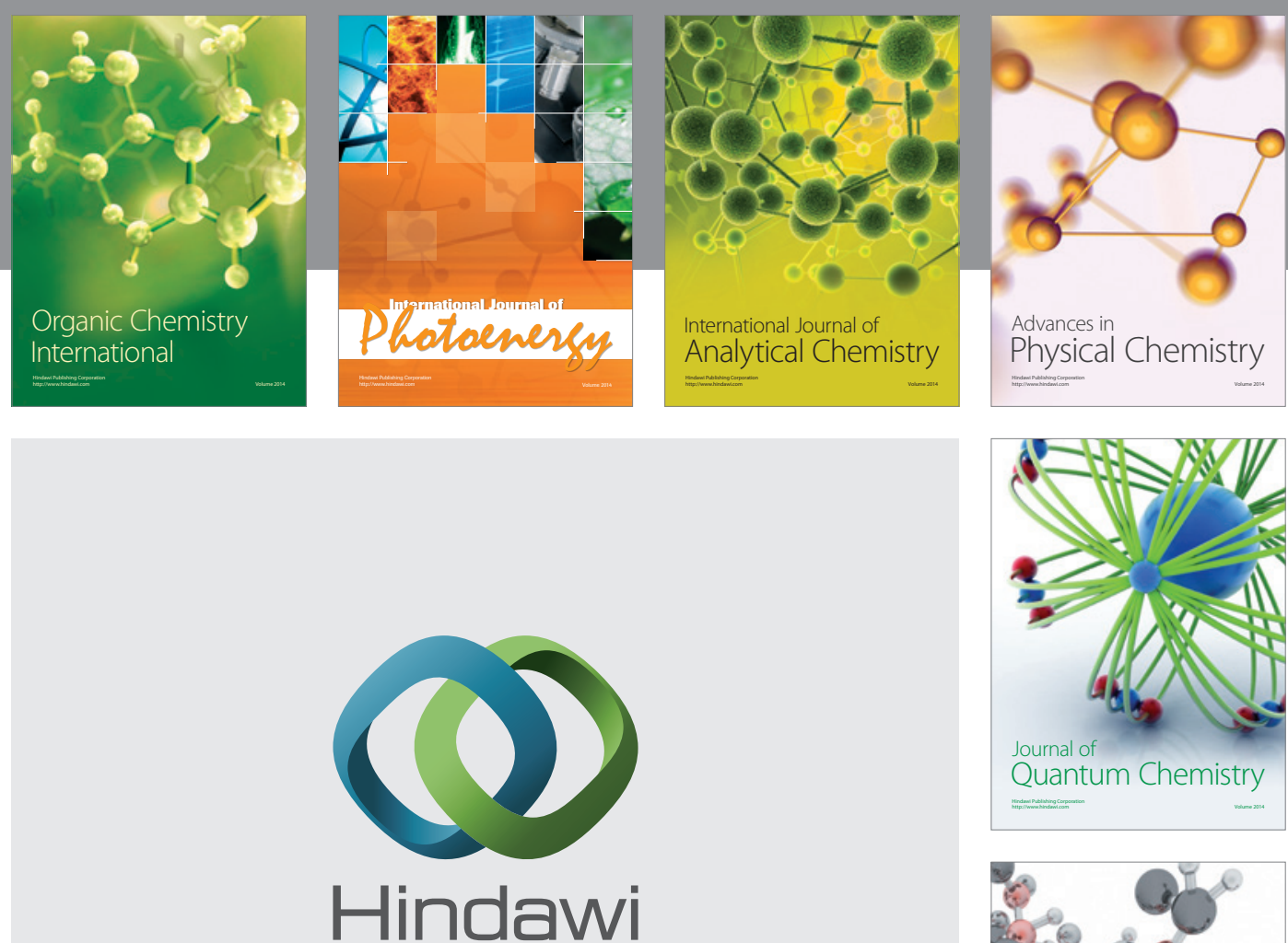

Submit your manuscripts at

http://www.hindawi.com

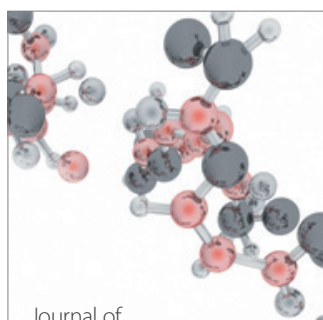

Analytical Methods

in Chemistry

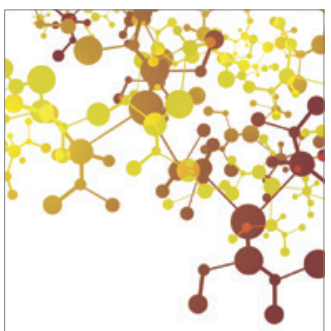

Journal of

Applied Chemistry

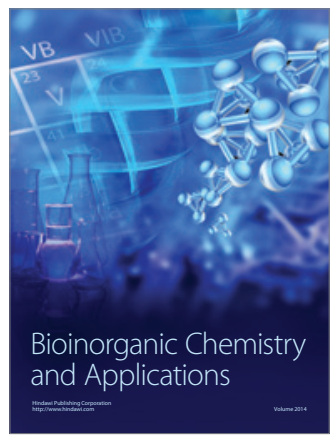

Inorganic Chemistry
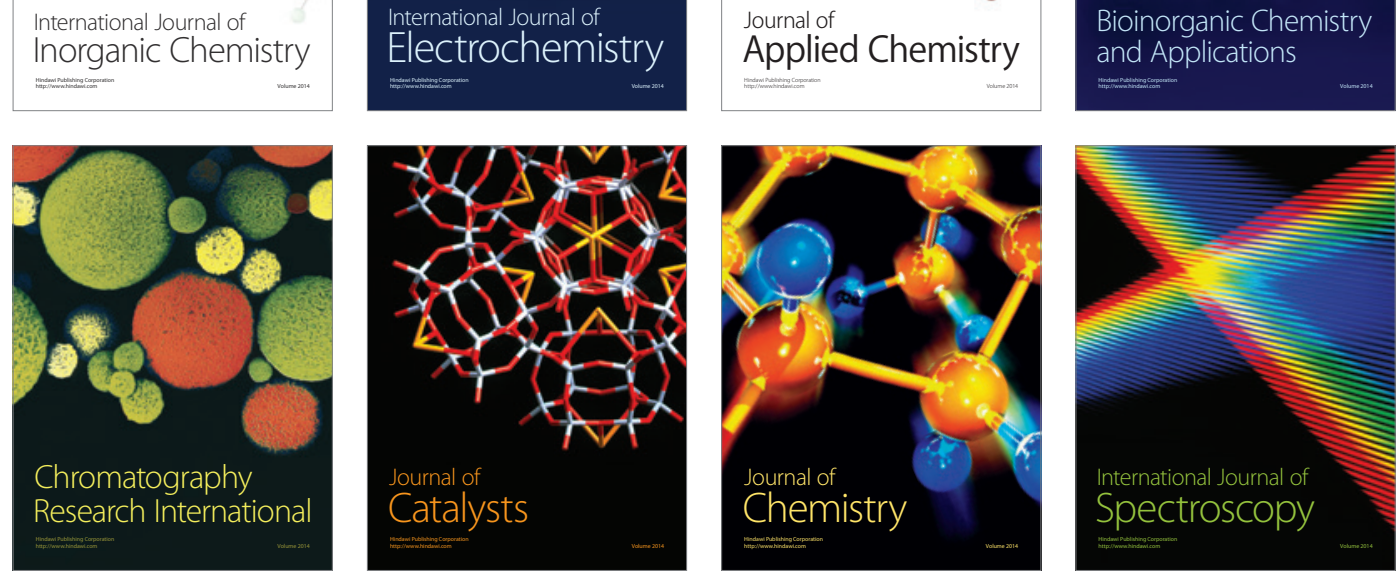